\title{
What can we tell about global auroral-electrojet activity from a single meridional magnetometer chain?
}

\author{
K. Kauristie ${ }^{1}$, T. I. Pulkkinen ${ }^{1}$, R. J. Pellinen ${ }^{1}$, H. J. Opgenoorth ${ }^{2}$ \\ 1 Finnish Meteorological Institute, Department of Geophysics, Helsinki, Finland \\ 2 Swedish Institute of Space Physics, Uppsala, Sweden
}

Received: 11 September 1995/Revised: 13 June 1996/Accepted: 25 June 1996

\begin{abstract}
The $A E$ indices are generally used for monitoring the level of magnetic activity in the auroral oval region. In some cases, however, the oval is either so expanded or contracted that the latitudinal coverage of the $A E$ magnetometer chain is not adequate. Then, a longitudinal chain in the key region would give more information of the real situation, but, of course, only during some limited UT-period. In order to find out the UT coverage of a single meridional chain, we have compared the global $A L$ and $A U$ indices with corresponding local indices determined using data from the meridional part of the EISCAT Magnetometer Cross during the years 1985-1987. A statistical study shows that the local indices are close (within relative error of 0.2) to the global $A U$ and $A L$ during periods $1500-2000$ UT ( $1730-2230$ MLT) and $2130-0130$ UT ( $\sim 0000-0400$ MLT), respectively. In the middle of these optimal MLT-sectors the EISCAT Cross sees more than $70 \%$ of the cases when the global $A E$ chain records activity. Then, also the correlation between the local and global indices is generally good $(>0.7)$. Thus we conclude that five to six evenly located meridional chains are needed for covering all the UTperiods. On the other hand, already the combination of IMAGE, CANOPUS, and the Greenland chains catches $\sim 50 \%$ of the substorms. Case-studies show that usually during 2130-1100 UT the $A L$ achieved from these chains reproduces the real $A L$ with good timing, although it does not follow all transient variations.
\end{abstract}

\section{Introduction}

The auroral-electrojet indices ( $A U, A L$, and $A E$, hereafter called the $A E$ indices) were designed in late 1960 s by Davis and Sugiura (1966) for routine-type monitoring of the

Correspondence to: K. Kauristie ionospheric currents in the auroral oval region. These indices are based on magnetic recordings of twelve observatories distributed along the auroral oval region (Fig. 1). $A U$ and $A L$ are the upper and lower envelope curves, respectively, of the superposed $H$-component variations (in intensity, but not in direction) observed at these stations, and $A E=A U-A L$ (Fig. 2). Before the superposition, the measurements are normalized by subtracting base-line values from the data. These base lines are determined for each station and for each month by averaging all $H$ values on the five internationally quiet days. Davis and Sugiura used 2.5-min resolution in their original work, but nowadays $A E$ indices are produced with 1-min resolution.

The ionospheric currents in the oval region are affected both by magnetopause-solar wind interaction processes and by energy release processes in the magnetotail. When energy and particles are transferred from the solar wind to the magnetosphere via dayside reconnection, usually both the eastward and westward auroral electrojets experience a smooth enhancement (directly driven activity). A part of the solar-wind energy and particles is stored into the magnetotail and dissipated later as abrupt bursts to the ionosphere, causing an extra strong westward electrojet (loading-unloading activity) to the midnight sector of the auroral oval (Baker et al., 1984).

Due to their relatively simple definition, the $A E$ indices are easy to relate both to their physical back-ground and to other physical quantities. $A U$ and $A L$ are generally assumed to express the strongest current densities of eastward (in the evening sector) and westward (in the morning sector) electrojets, respectively. Electrojet activity, however, is latitudinally limited and thus the $A E$ indices are reliable only if the contributing $A E$ station happens to be at the right latitudes.

During substorms, both directly driven and loadingunloading activity are present in the $A E$ indices. $A U$ increases smoothly during the substorm growth and expansion phases and decays back to quite-time values during the recovery phase, while $A L$ shows more dynamic 


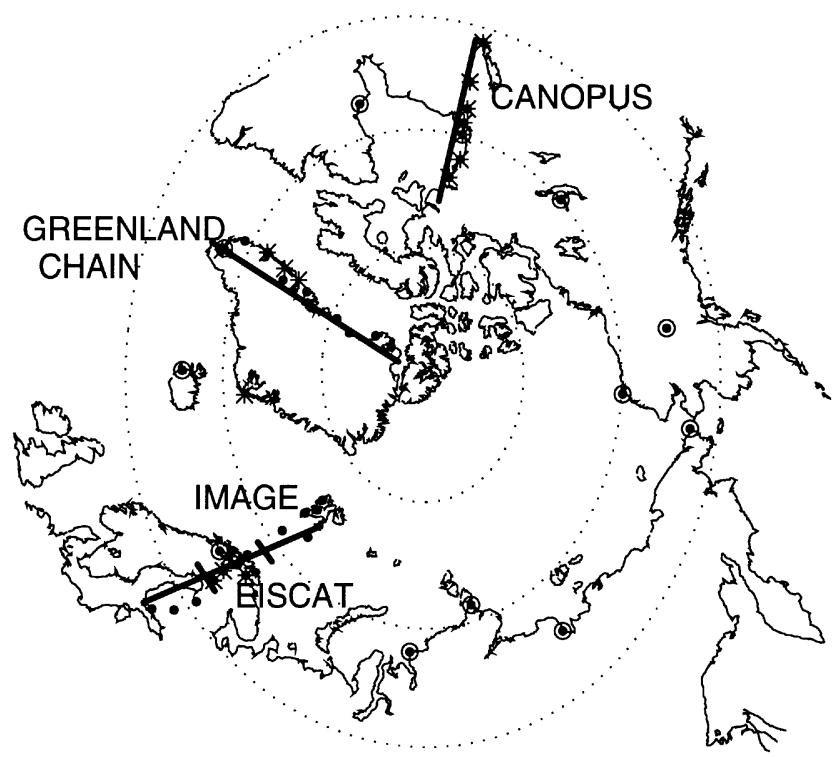

$\mathrm{AE}-\mathrm{CHAIN}=$

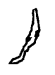

Fig. 1. The $A E$ stations and the IMAGE/EISCAT (EISCAT is the shorter part within the tick marks in the middle of the IMAGE chain), CANOPUS, and Greenland magnetometer chains. The stations used in the case-studies are marked with asterisks. The dotted lines show the corrected geomagnetic latitudes 60,70 , and 80 behavior, especially during the expansion phase, which typically starts with an abrupt $A L$ drop (Fig. 2; Caan et al., 1978; Kamide and Kroehl, 1994).

From the viewpoint of statistical studies the $A E$ indices are superior to other activity indices. In addition to their physically relevant definition, they cover a significant part of the interesting oval region, have adequate time-resolution, are easily available, and long time-series exist. Thus they have been used in many studies concerning, e.g., the solar wind-magnetosphere interaction (Bargatze et al., 1985; Baker et al., 1990; Shan et al., 1991; Takalo et al., 1993) and substorms (Caan et al., 1978; Kamide and Kroehl, 1994; Pellinen et al., 1994; Weimer, 1994; Kauristie, 1995).

According to Kamide and Akasofu (1983), the accuracy of the $A E$ indices reduces when magnetic activity decreases below $250 \mathrm{nT}$. Then the oval is so contracted that the $A E$ stations cannot monitor it properly. Similarly, during strong activity the oval expands to such low latitudes that the $A E$ chain cannot follow the electrojet activity either. Furthermore, during continuously enhanced activity (e.g., steady convection periods), the oval can be much wider than it usually is, also leading to possible misinterpretations of the activity level if only $A E$ indices are used (Sergeev et al., 1996). In such cases a meridional chain is necessary for discriminating spatial effects from real temporal behavior. The longitudinal coverage of a single meridional chain is, however, limited.

\section{The global AE-chain:}

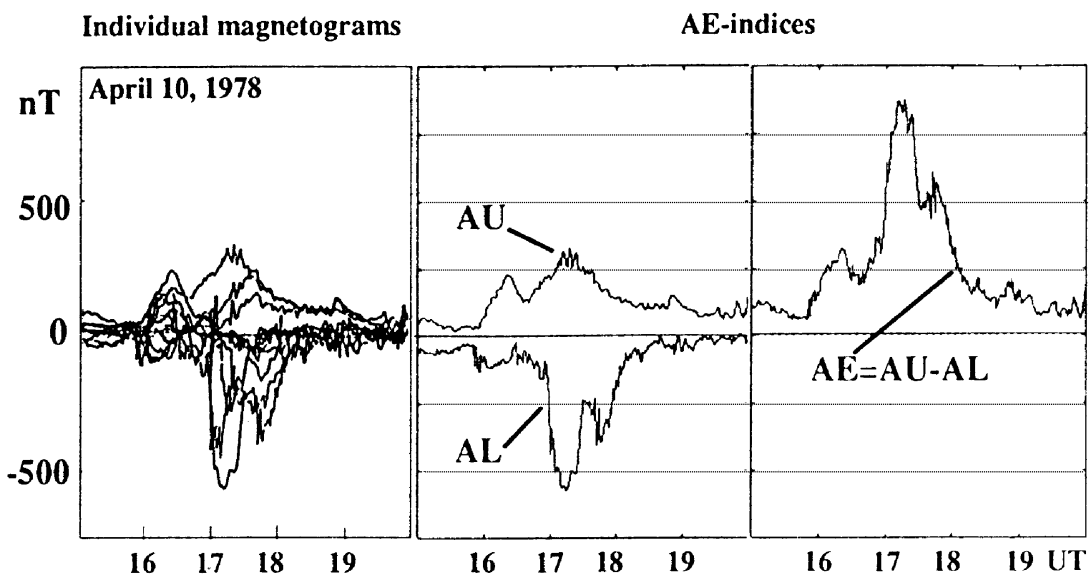

The EISCAT Magnetometer Cross:
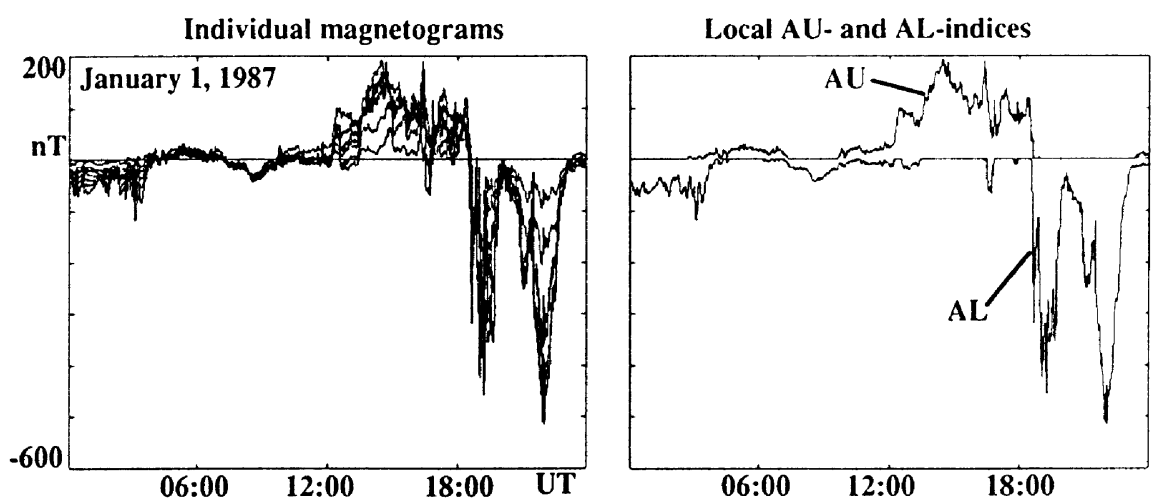

Fig. 2. Construction of the $A E$ indices. Upper panel: the global $A E$ indices. Lower panel: the local quasi- $A U$ and $A L$ indices 
Questions like what are the activity level and the longitudinal (or UT) coverages of a single chain, or which UT-periods can be covered with the present operating meridional chains, are important now during the ISTP (International Solar Terrestrial Physics) program, as information on the global magnetic activity level is required in a rapid time-scale after the satellite observations (e.g., from WIND, POLAR, GEOTAIL, and INTERBALL). New preliminary oval indices (resembling $A L$ index) based on data from some automated stations are already available (see the World Wide Web at http://wdcc1.bnsc.rl. ac.uk/gbdc/auroralovals.html). There are three indices monitoring activity in contracted, standard (at the latitudes of $A E$ stations), and expanded oval. The coverage of the indices is improved continuously as data from new stations are obtained. In addition to magnetic recordings data from radars and optical instruments are also needed to support the satellite measurements which provide highresolution data, but suffer from spatial-temporal ambiguity. The ground-based observations help in placing the in situ observations in a wider context and provide important information of the ionospheric boundary conditions.

We compare the global $A L$ and $A U\left(A L_{g l o}\right.$ and $\left.A U_{g l o}\right)$ indices with corresponding local quasi-indices $\left(A L_{l o c}\right.$ and $\left.A U_{\text {loc }}\right)$ determined using the recordings of the EISCAT Magnetometer Cross in northern Scandinavia and Finland. In Sect. 2 we describe the local data set and the parameters used for characterizing the differences between the local and global indices. The optimal UT-periods when the local indices give relevant information of the global activity are defined in Sect. 3. The activity dependence of these UT-periods is analyzed as well. In Sect. 4 the common coverage of the EISCAT/IMAGE, Greenland, and CANOPUS chains is studied during two 1-week test periods. Section 5 comprises the conclusions.

\section{Data analysis}

The local $A E$ indices are based on the recordings of the EISCAT Magnetometer Cross (c.f. Fig. 1; Lühr et al., 1984) during the years 1985-1987. This cross consists of a main meridional chain (five stations) around geographic longitude $\sim 23^{\circ}$ and two side stations $\sim 3^{\circ}$ to the west and east of the main chain. The chain covers magnetic latitudes $63^{\circ}-67^{\circ}$ (here the magnetic coordinates are by Baker and Wing, 1989). We had to omit the recordings of KIL (the western-side station) from the analysis because of technical problems during 1985, but fortunately this does not cause any significant limitations to the longitudinal coverage of the local indices.

The local $A E$ indices were constructed with exactly the same procedure as the global $A E$ indices. The base-line values were defined for each month using the average $H$-component values of the five internationally quiet days $\left(H=\sqrt{X^{2}+Y^{2}}\right.$, where $X$ and $Y$ are the geographic north and east components of the magnetic field). After subtracting the base lines the $H$-components of the six stations were superposed and the envelope curves were used as local $A U$ or $A L$ indices, when positive or negative, respectively (cf. Fig. 2). Located at one fixed longitude, the EISCAT Cross usually gives either $A U$ or $A L$. However, sometimes close to the Harang discontinuity or during quiet periods the upper envelope curve can be positive and the lower curve negative.

The differences between the local and global indices can be characterized by computing the average relative error, $E(t)$, which is defined, e.g., for $A L$, as

$E_{A L}(t)=\frac{A L_{g l o}(t)-A L_{l o c}(t)}{A L_{g l o}(t)}$,

where $t$ is the time (with 1-min resolution). $A L_{g l o}$ and $A L_{l o c}$ are negative and thus $E_{A L}$ is negative when the local chain sees stronger activity than the global one (i.e., when $A L_{l o c}<A L_{g l o}$, but $\left.\left|A L_{l o c}\right|>\left|A L_{g l o}\right|\right)$. In this case the deviation is due to the improvement in the local index when compared to the global one. When the local chain is outside the key-region, $\left|A L_{l o c}\right| \sim 0$ and $E_{A L} \sim 1$. These limit values are valid also for $E_{A U}$ when defined similarly as $E_{A L}$. When computing the relative errors the pairs of local and global values were categorized according to magnetic activity and binned to 1-h UT bins. The 1-h averages are used for defining the optimal UT-periods when the relative errors are below 0.2 , which we consider as a suitable tolerance level for global activity monitoring. Our definition for the relative error does not take into account the periods when $A L_{g l o}>0$ or $A U_{g l o}<0$. Such periods, however, are rare and thus not statistically significant.

In order to estimate the quality of the local indices further, we computed the Pearson correlation coefficients, $C(t)$, between the two data sets:

$$
\begin{aligned}
C_{A L}(t)= & \sum_{i=1}^{n}\left(A L_{g l o}^{i}(t)-\bar{A} L_{g l o}(t)\right)\left(A L_{l o c}^{i}(t)-\bar{A} L_{l o c}(t)\right) \\
& \times\left(\sum_{i=1}^{n}\left(A L_{g l o}^{i}(t)-\bar{A} L_{g l o}(t)\right)^{2}\right. \\
& \left.\times \sum_{i=1}^{n}\left(A L_{l o c}^{i}(t)-\bar{A} L_{l o c}(t)\right)^{2}\right)^{-1 / 2},
\end{aligned}
$$

where $t$ is UT (with 10-min resolution), the summation $\sum_{i=1}^{n}$ goes over index values recorded at the moment $t$, and $A L_{g l o}(t)$ and $\bar{A} L_{l o c}(t)$ are the mean values at each UT. We computed $C_{A L}$ and $C_{A U}$ for four different activity levels. The data were binned as whole events (where events are the local and global time-series observed during 24-h UT-periods centered at midnight for $A L$ and at noon for $A U$ ), not as individual points as in the case of relative errors. The binning was done according to the minimum $(A L)$ and maximum $(A U)$ 1-min values of the events.

Press et al. (1992) present a method for estimating the statistical significance of the correlation coefficients. For large data sets (including hundreds of data points) the correlation coefficient can be considered as a random variable with normal distribution. In the null hypothesis the correlation is assumed to be zero (i.e., the mean value of the normal distribution is zero). The probability of achieving at least the observed correlation value is 
computed with the complementary error function

$S C_{A L}(t)=\frac{2}{\sqrt{\pi}} \int_{\left(\left|C_{A L}(t)\right| \sqrt{n}\right) / \sqrt{2}}^{\infty} \exp \left(-u^{2}\right) d u$,

where $n$ is number of the observations at the considered $t$. If $S C_{A L}(t)$ is small (below 0.01 is a commonly used limit), the null hypothesis can be rejected and the correlation can be considered as significant. The optimal UT-periods are defined as periods when significant correlation is larger than 0.7 .

\section{Comparison between the global and local $A U$ - and $\boldsymbol{A L}$-indices}

\subsection{How often and how well does the local chain record activity?}

In order to see what are the key regions of the $A U$ and $A L$ activity, we estimated the probability (as a function of UT) of the local chain to record activity when the global chain records it. This was done by comparing 20 -min averages of the global and local indices. The local chain was defined to record the global activity when the absolute values of both the averages exceeded $100 \mathrm{nT}$. Then the number of local activity observations (at each $t$ ) was divided by the number of global activity observations. The results are shown in Fig. 3. The EISCAT Cross sees $\geq 70 \%$ of the $A U$ activity events during 1430-1700 UT and the $A L$ activity events during 2130-0100 UT. During these UTperiods the EISCAT Cross scans the MLT-sectors $\sim 1700-1930$ and $\sim 0000-0330$, respectively (hereafter we use only UT-periods from which the approximate MLT-sectors are obtained by adding $2.5 \mathrm{~h}$ ).

The UT dependence of the relative errors $E_{A L}$ and $E_{A U}$ are shown in Fig. 4. The average relative errors are smallest during 1500-2000 UT (for $A U$ ) and 21300130 UT (for $A L$ ). In these regions the meridional chain gives a representative estimate of the global activity level. To summarize, Figs. 3 and 4 show the time-periods during each day when, one, the probability of observing a disturbance is high (Fig. 3) and two, the relative error between the locally measured activity and the activity recorded by the global chain in small (Fig. 4).

\subsection{Dependence on the global and local activity levels}

The relative errors of Eq. 1 depend also on the intensity of the activation: stronger events are easier to record with a local chain. Figure 5 shows how $E_{A L}$ behaves at different activity levels, when the data have been binned according to local activity. The pairs of data points were categorized into four groups: $A L_{l o c}<-600 \mathrm{nT}, \quad-600 \mathrm{nT} \leq$ $A L_{l o c}<-300 \mathrm{nT}, \quad-300 \mathrm{nT} \leq A L_{l o c}<-100 \mathrm{nT}$, and $A L_{\text {loc }} \geq-100 \mathrm{nT}$. The average error curves show the time-periods when the local chain records activity at the given level, and how well in these cases the observed activity describes the global activity. The average error curve for the strongest $A L$ activity level shows that the
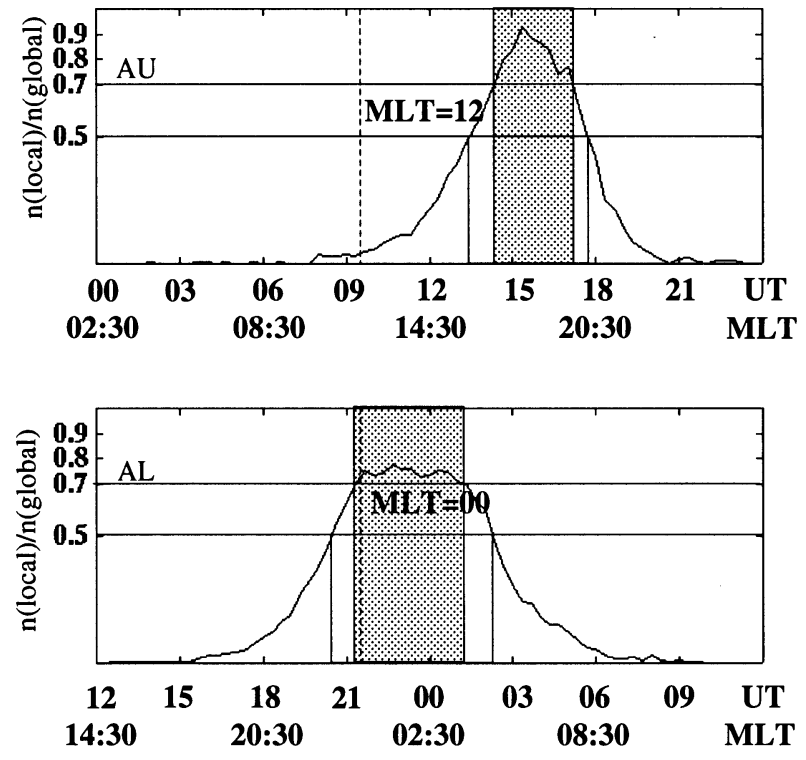

Fig. 3. Distribution of the local observations of $A U$ (upper panel) and $A L$ (lower panel) activity as functions of UT (or MLT). The gray shadings mark the UT-periods when the EISCAT Cross catches at least $70 \%$ of the activity events which the $A E(12)$ chain records (for more details, see text)
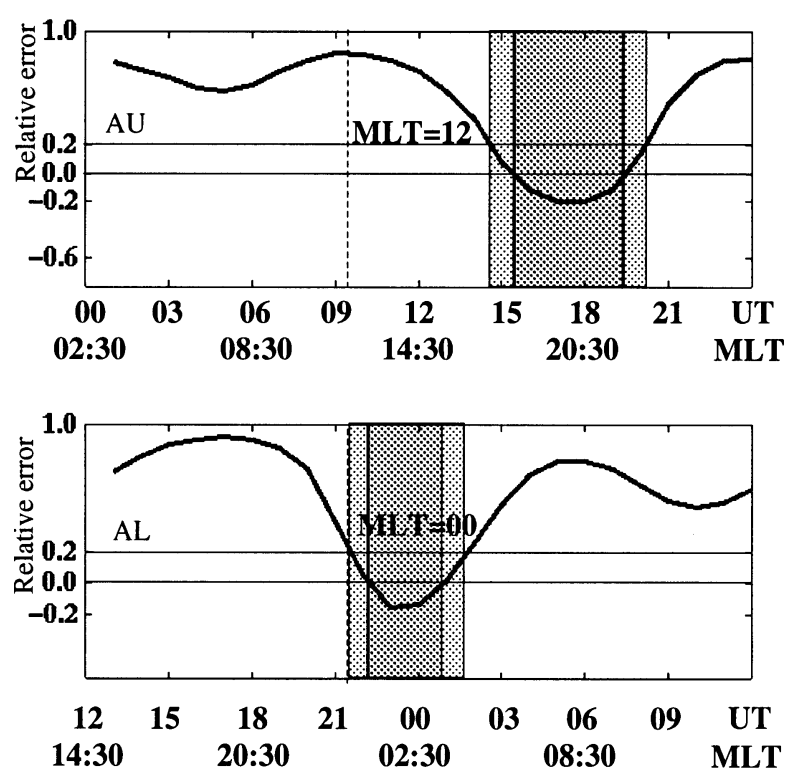

Fig. 4. UT dependence of the average relative errors between the global and local $A U$ (upper panel) and $A L$ (lower panel). The UTperiods when the error is below 0.2 and below 0 (i.e., the local chain sees stronger activity than the global one) are marked with lighter and darker shadings, respectively

local chain records activity below $-600 \mathrm{nT}$ during 1730-0630 UT and then usually also the global chain sees approximately the same activity. In addition, during 1800-0600 UT, $E_{A L}$ is negative and thus the local chain records stronger activity than the global one. At the next two activity levels the local chain records activity during a wider UT-period, but this activity is close to the global index during periods 1800-0400 UT and 2000-0300 UT, 

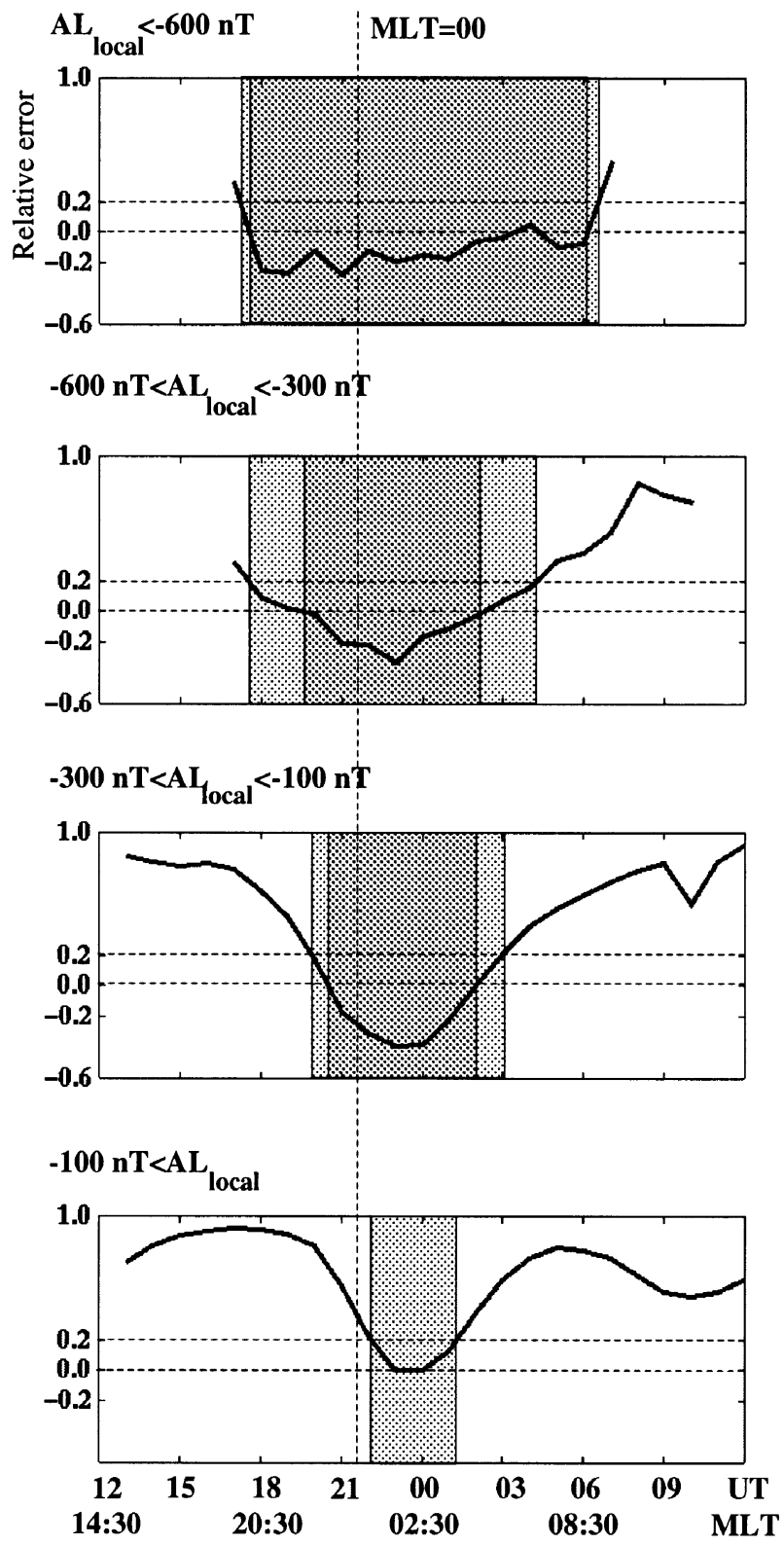

Fig. 5. Activity dependence of the average relative errors between the global and local $A L$. The data have been binned according to local activity. The UT-periods when the error is below 0.2 and below 0 (i.e. the local chain sees stronger activity than the global one) are marked with lighter and darker shadings, respectively

respectively. $E_{A L}$ at the lowest activity level shows just that if the local chain cannot see any activity during period 2200-0100 UT then it is really quiet. The narrow time window in this case also shows that small events are easy to miss when not exactly at the correct local timesector.

Figure 6 presents the activity dependence of $E_{A U}$ (for levels $A U_{l o c}<100 \mathrm{nT}, 100 \mathrm{nT} \leq A U_{l o c}<300 \mathrm{nT}, 300 \mathrm{nT}$ $\leq A U_{l o c}<500 \mathrm{nT}$, and $\left.A U_{l o c} \geq 500 \mathrm{nT}\right)$. At the activity level $100 \mathrm{nT} \leq A U_{l o c}<300 \mathrm{nT}$ in almost all UT bins, the local chain records stronger $A U$ activity than the global chain. However, the number of data points is small in bins other than 1400-1800 UT. At the three other activity

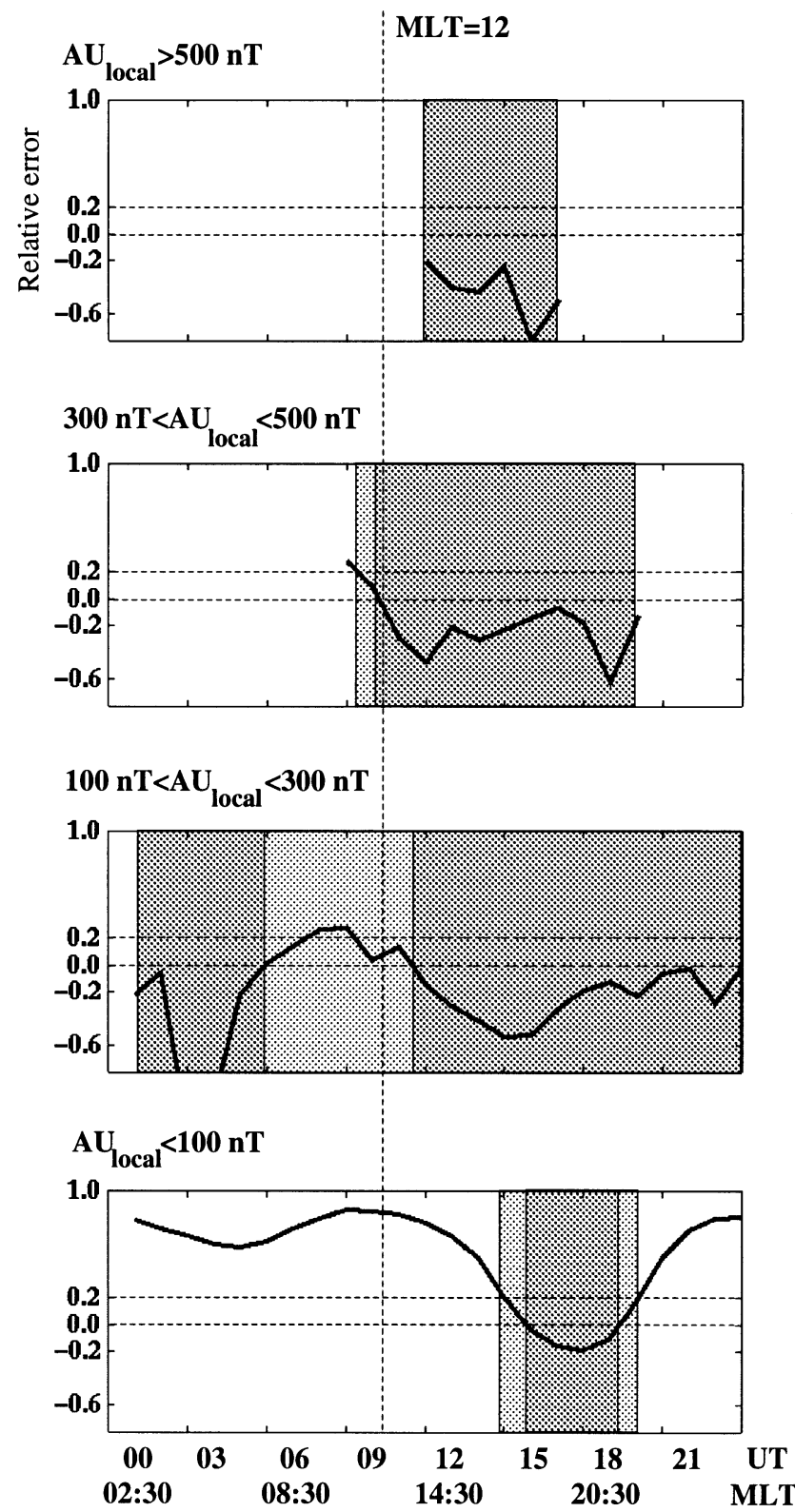

Fig. 6. Activity dependence of the average relative errors between the global and local $A U$. The data have been binned according to local activity. The UT-periods when the error is below 0.2 and below 0 (i.e. the local chain sees stronger activity than the global one) are marked with lighter and darker shadings, respectively

levels the local and global $A U$ are close to each other around 0900-2000 UT, depending strongly on the activity level. The optimal UT-period of $A U$ does not show as systematic activity dependence as that of $A L$, which shortens as the local activity decreases. The wide local time-sectors of negative $E_{A U}$ (especially at the level $\left.100 \mathrm{nT} \leq A U_{l o c}<300 \mathrm{nT}\right)$ can be partly due to base-line problems, but they also show the latitudinal limitations of the $A E$ chain: the evening-sector eastward electrojet contributing to $A U$ is at lower latitudes than the morningsector westward electrojet, which contributes to $A L$ (Rostoker and Phan, 1986). Eleven stations of the $A E$ chain are at higher magnetic latitudes than the most southern 
station of the EISCAT Cross. These stations do not monitor the eastward electrojet as well as the local chain (i.e., $\left.E_{A U}<0\right)$. On the other hand, during 0600-1200 UT, when the southernmost $A E$ stations Tixie Bay and Cape Wellen (magn. lat. $61^{\circ}-62^{\circ}$ ) are in the evening sector $(\mathrm{MLT} \sim 1800-2400)$ and contribute to global $A U, E_{A U}$ is positive.

When the data are binned according to global activity (with the same bins as in Fig. 5), $E_{A L}$ shows nearly similar behavior as in Fig. 4 (being below 0.2 during 22300130 UT at the highest activity level and during 22000200 UT at the other levels). $E_{A U}$ depends on the activity level so that the optimal period shifts to earlier UT hours as the activity increases (being 1430-2030 UT, 1430-1730 UT, 1300-1700 UT, and 1400-1500 UT for the levels $A U_{\text {glo }}<100 \mathrm{nT}, 100 \mathrm{nT} \leq A U_{g l o}<300 \mathrm{nT}$, $300 \mathrm{nT} \leq A U_{g l o}<500 \mathrm{nT}$, and $A L_{g l o} \geq 500 \mathrm{nT}$, respectively). The average error curves for the global binning are different (not reproduced here), because in the local binning also the rare data points outside the key regions are very pronounced, while in the global binning they do not have any statistical significance. As a final conclusion from Figs. 4-6, it can be said that when the local chain records significant activity it yields a better representation of the activity than the global index, but on the other hand only when the local chain is in the key regions of $A U$ and $A L$ (c.f. Fig. 3) we can be sure that it catches the major part of the activations.

\subsection{The correlation coefficients}

The correlation coefficients give a somewhat different view to our problem. The average relative error between two variables can be small even though they behave in a different manner. Figure 7 shows examples of $C_{A U}(t)$ and $C_{A L}(t)$, which are based on data binned according to local activity. The optimal periods defined from $C_{A U}(t)$ and $C_{A L}(t)$ are generally shorter than those in Figs. 5 and 6 (at the highest activity levels), but longer than in Fig. 4. Here again, the same activity levels were used as in the relative error computations. The best correlation is achieved at the highest $A L$ activity level, while at the lowest activity levels $C_{A U}$ and $C_{A L}$ did not exceed 0.7 at all. $C_{A L}(t)$ is above 0.7 during periods 2100-0500 UT, 2030-0600 UT, and 2200-0530 UT for the three highest activity levels, respectively. For $A U$ these periods at the second and third activity levels are somewhat shorter: 1200-1630 UT and 1500-1700 UT. In the bin of highest $A U$ activity level there were too few points for meaningful correlation computations. In the other cases the number of data point pairs varied from 50 to 300 .

\section{Discussion}

\subsection{Obtaining a global activity index from several, evenly separated chains}

With the results just presented we are able to estimate the common coverage of the IMAGE (Lühr, 1994), Greenland
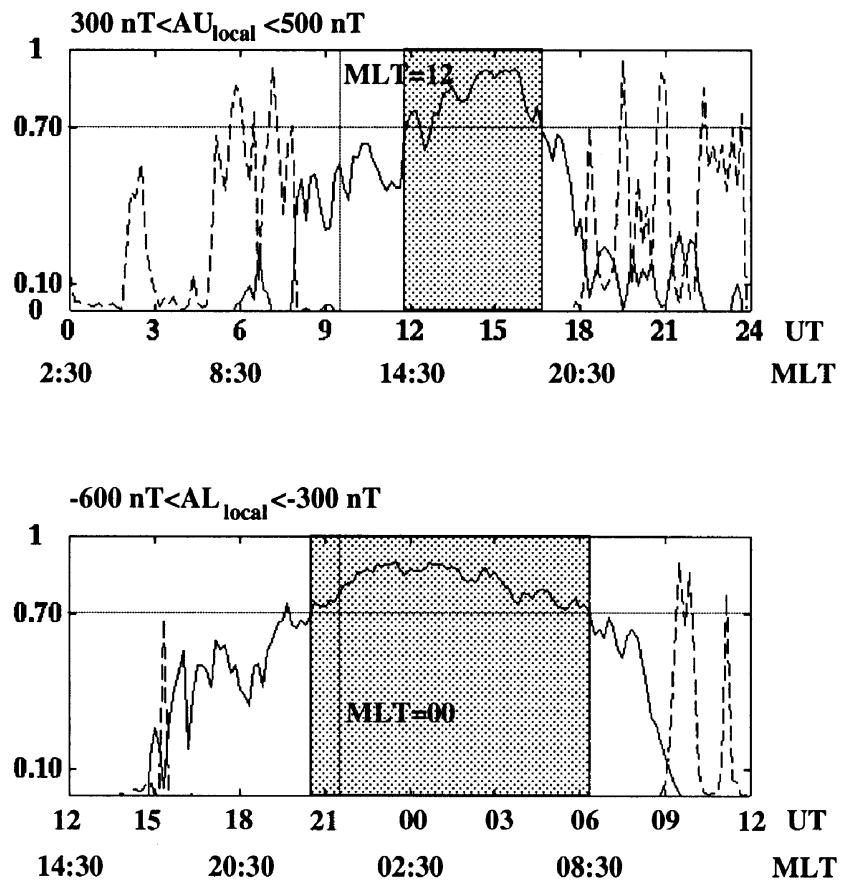

Fig. 7. Correlation (solid line) and an estimate for its statistical significance (dashed line) between the global and local $A U$ (upper panel) and $A L$ (lower panel) indices. The correlation can be considered as significant when the dashed line is below 0.01. The gray shadings show the UT-periods when the correlation is better than 0.7

(Friis-Christensen et al., 1985), and CANOPUS (Samson et al., 1991) chains (see Fig. 1). These fully automated chains could serve as the primary data sources for providing a quasi- $A L$ index only within a few weeks' time-delay. Assuming that the average error curves would be similar also for the other two chains, the joint coverage of the three chains would be around $14 \mathrm{~h}$ (2130-1100 UT).

This result was tested by constructing $A U$ and $A L$ for two 1-week periods (22-28 February and 18-24 March 1991) using the recordings of 17 stations (marked with stars in Fig. 1) from the three chains. Figure 8 shows the constructed $A L\left(A L_{17}\right)$ together with the real $A L$ index during 5 days from our test periods. The UT-range which, according to our statistical analysis, is beyond the reach of the 17 stations, is marked with the vertical bars. Elsewhere, the longitudinal distribution of the stations differs from the $A E$ chain (which during the studied periods consisted of 13 stations having essentially the same longitudinal distribution as the official $A E$ (12) chain) only with the one $A E$ station between the Greenland and CANOPUS chains. Consequently $A L_{17}$ reproduces the real $A L$ with good timing (i.e., without significant delays in the abrupt drops), although it does not follow all the variations. The transient differences in the intensity are most probably due to local intensifications which cannot be monitored identically with the two different magnetometer networks.

Both the average error analysis and the correlation coefficients between $A L_{l o c}$ and $A L_{g l o}$ show that when even in the late morning sector $(\sim 0400$ MLT), the meridional 


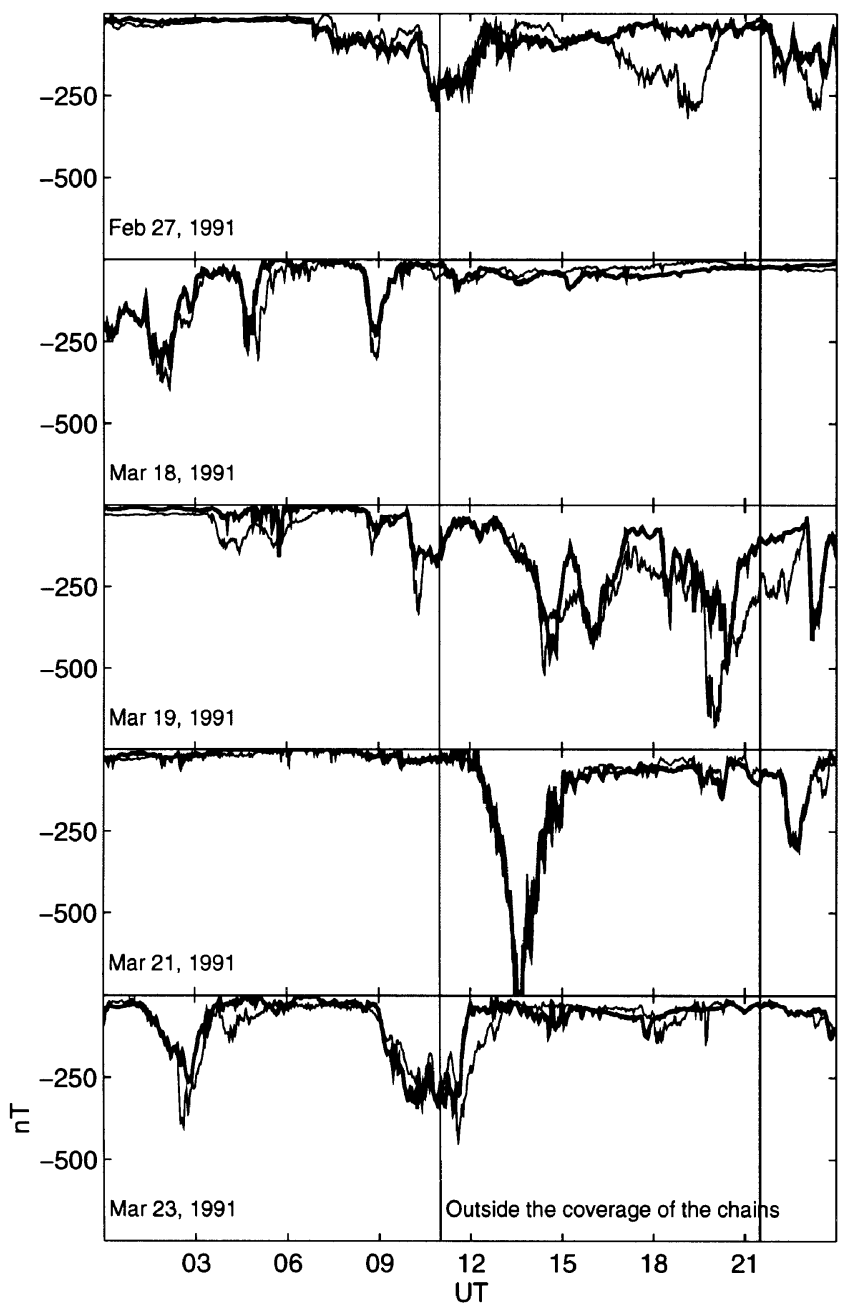

Fig. 8. $A L_{17}$ (heavy solid line) and the real $A L$ index (thin solid line) during 5 days from February-March 1991

chain provides a representative estimate for the global $A L$. Especially during the substorm recovery phase the activity concentrates to morning sector (Kamide and Kroehl, 1994). For example, on 19 March around 2000 UT (third panel in Fig. 8), all the chains were in the evening sector, and consequently $A L_{17}$ failed to estimate the recoveryphase activity although it took place during the optimal UT-periods. On the other hand, on 21 March during 1200-1500 UT (fourth panel in Fig. 8) an intensive, but relatively short activation took place. Although all the 17 stations were in late morning sector ( $\sim 0600 \mathrm{MLT}), A L_{17}$ gave a good estimate for $A L$ both in timing and in intensity during the whole substorm.

Thus on average, with the three meridional chains, $\sim 50 \%$ of the global activity can be covered. With the addition of a few stations in the Russian and/or Alaskan time-sectors would complete the circle to cover almost all local times. In such a case, with the longitudinal coverage of the chains, much more information could be extracted than is today possible with the $A E$ chain. In local studies the chain closest to the key region could be used, e.g., for estimating the location and width of the auroral electrojet. However, we emphasize that applying the error curve of
Fig. 4 to Greenland or CANOPUS chains gives only an estimate of the real error curve which can show some differences in behavior. Furthermore, the IMAGE chain is longer (covering magnetic latitudes $\sim 57^{\circ}-76^{\circ}$ ) than the former EISCAT chain. Thus the new chain has a better activity coverage, but perhaps also slightly widened UT coverage, than the old chain.

\subsection{Improving the predictions with a linear filtering method?}

We tried to improve the predictions of global activity from the local measurements by computing a linear filter between the local and global $A L$ indices. The goal was to find a filter which gives $A L_{g l o}$ when only $A L_{l o c}$ is known, and the meridional chain is slightly outside its optimal MLT-region. A linear filter predicts $A L_{g l o}$ at the given time $t$ using $A L_{l o c}$ values preceding and following that time, i.e., $A L_{g l o}\left(t_{i}\right)=\sum_{k=-d_{1}}^{d_{2}} a_{k} A L_{l o c}\left(t_{i+k}\right)+E\left(t_{i}\right)$, where $a_{k}$ are the filter coefficients, $t_{i}$ is the considered movement (ith time-step from the beginning of the optimal UTperiod), and $d_{1}$ and $d_{2}$ are parameters defining the number of preceding and following local values taken into account in the prediction. $E\left(t_{i}\right)$ is the error between the predicted $A L_{q l o}\left(t_{i}\right)$ and the real $A L_{g l o}\left(t_{i}\right)$. The coefficients $a_{k}$ can be defined by minimizing the total sum (over all times and events) of the squared errors.

We tested the linear prediction method with 5-min average values of the $A L$ events occurring at 2000 0400 UT. We concentrated on events where the minimum local $A L$ value occurred between 0130 and 0400 UT. The local values $50 \mathrm{~min}$ before and $75 \mathrm{~min}$ after the considered moment were taken into account in the prediction (i.e., $d_{1}=10$ and $d_{2}=15$ ). The main difficulty was to find suitable criteria for categorizing the events. If the filter is defined using a group of very different events, all the other coefficients except that for $k=0$ smear out. Activity binning according to the local median values appeared to produce the most homogeneous subgroups for this purpose (two groups: medians $-100--30 \mathrm{nT}$ and medians below $-100 \mathrm{nT}$ ). The total amount of events was 52, 26 of which were used for defining the filter and 26 for testing it. In 17 cases of the 26 test events the predicted curve gives somewhat better estimate for $A L_{g l o}$ than $A L_{l o c}$ during 0100-0300 UT. Improved predictions were more common in the lower activity bin (11 of 15 cases) than in the higher activity bin (6 of 11 cases).

Figure 9 shows one of the best examples of the achieved predictions. Although in this case the intensity of the predicted $A L_{g l o}$ is close to the real $A L_{g l o}$, generally the linear filtering did not improve $A L_{l o c}$ values significantly. Our poor success can be partly due to the unfavorable distribution of the $A E$ stations. For the CANOPUS chain the predictions could be better, because there the nearby $A E$ stations are suitably located for this purpose (Postede-la-Baleine and Yellowknife, $\sim 30^{\circ}$ to the east and west, respectively), while the nearby stations of EISCAT Cross are either too close (Abisko, $4^{\circ}$ to the west) or too far (Dixon Island, $49^{\circ}$ to the east). 


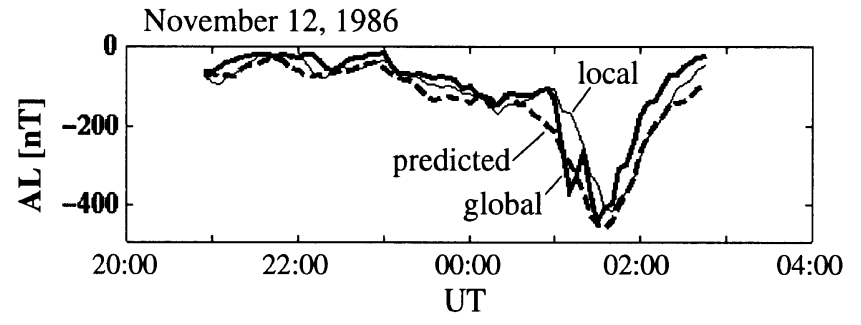

Fig. 9. An example on predicting the $A L$ index. The predicted curve has been obtained from the local $A L$ indices by linear filtering

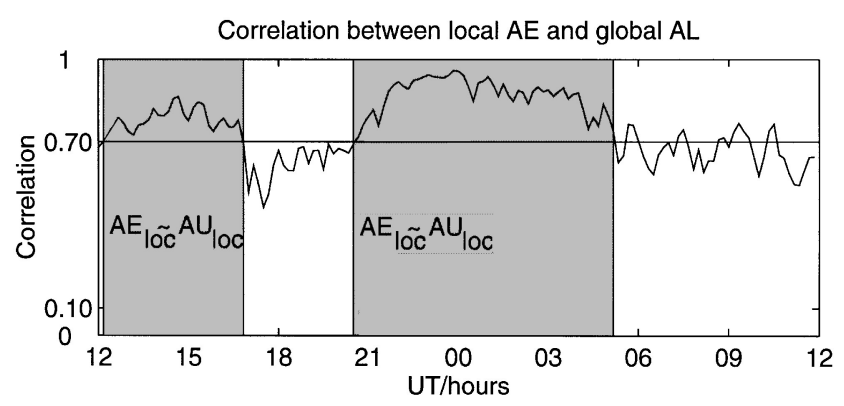

Fig. 10. Correlation between the global $A L$ and local $A E$ when maximum 1-min local $A E>500 \mathrm{nT}$. The gray shadings show the UT-periods when the correlation is better than 0.7

\subsection{Correlation between the local AE and the global AE: possible evidence of eastward-electrojet enhancement during substorms}

Figure 10 shows the correlation (computed using 136 pairs of data points at each UT-moment) between the $A E_{l o c}$ and $A L_{g l o}$ when maximum 1-min $A E_{l o c}>500 \mathrm{nT}$. During 1430-1700 UT, $A E_{l o c} \sim A U_{l o c}$ (because then $A L_{l o c} \sim 0$ and $A U_{l o c} \neq 0$, c.f. Fig. 3) and during 2130-0100 UT, $A E_{l o c} \sim\left|A L_{l o c}\right|\left(A U_{l o c} \sim 0\right.$ and $A L_{l o c} \neq 0$, c.f. Fig. 3). Around both these periods the correlation is clearly higher than elsewhere. For the nightside this is not surprising, but according to Fig. 10 the $A U$ activity in the MLT sector $\sim 1430-1930$ is closely related to the $A L_{g l o}$ activity, too. If a significant part of the $A L_{g l o}$ activity is assumed to be due to loading-unloading processes, our result suggests that also $A U$ variations correlate well with substorm activity. This could be due to the so-called eastward explosive electrojets. Grafe (1994) shows examples where enhancements of the eastward electrojet are accompanied by enhanced electron precipitation and $\mathrm{Pi} 2$ pulsations, i.e., phenomena which are usually related to the substorm current wedge in the midnight sector.

The explosive eastward electrojets are usually more dynamic and at lower latitudes than the convection-type electrojets (Grafe, 1994), and thus the EISCAT Cross (when in the right position) monitors them better than the $A E$ chain. In our analysis, the most active $A U$ region shifts to earlier MLT values when activity level is increasing. Combining this information with the schematic Fig. 10 of Grafe (not shown here) suggests that the possible observations of explosive eastward electrojets concentrate to the events of our highest activity bin.

\section{Conclusions}

We have made comparisons between the global $A U$ and $A L$ indices and corresponding quasi-indices determined from the recordings of the meridional part of the EISCAT Magnetometer Cross. Our statistical studies reveal that the main part of the local $A U$ and $A L$ values are achieved when the meridional chain scans the MLT-sectors 1700-1930 and 0000-0330, respectively. Then the local chain catches $70 \%$ of the cases when the global chain sees activity. In MLT-sectors 1730-2230 (for $A U$ ) and 0000-0400 (for $A L$ ), the strength of the observed local activity is on average more than $80 \%$ of the simultaneous global activity (c.f. Fig. 11), but especially in the middle of these optimal MLT-sectors the local indices usually show stronger activity than the global ones. Thus the meridional chain monitors better the activity in the expanding oval than a single $A E$ station.

The optimal MLT-sector of $A L$ activity does not show any significant dependence on global activity level (but stays around $\sim 0030-0430$ MLT), while that of $A U$ activity shifts to later MLT values as the activity decreases (1630-1730 MLT when $A U_{g l o}>500 \mathrm{nT}$ and 1700 $2300 \mathrm{MLT}$ when $\left.A U_{g l o}<100 \mathrm{nT}\right)$. Binning according to local activity shows that in those rare cases when strong activity outside the optimal MLT-sectors is observed, it is usually stronger than in the global indices.

In the optimal MLT-sectors the correlation coefficient between the local and global indices is generally higher than 0.7. The correlation coefficient is at this level also for one or two MLT hours before (for $A U$ ) or after (for $A L$ ) the optimal periods. Thus in these sectors the local indices follow the behavior of the global indices, although their variations are not strong.

We tested the possibility of improving the longitudinal coverage of the EISCAT Cross by defining a linear filter between $A L_{g l o}$ and $A L_{l o c}$. Such a filter could be used for predicting $A L_{g l o}$ when only $A L_{l o c}$ is available. However, due to the great variability in the location and spatial scales of substorm activity, finding representative filters was difficult. Thus no significant improvement in the UT coverage was achieved with this method.

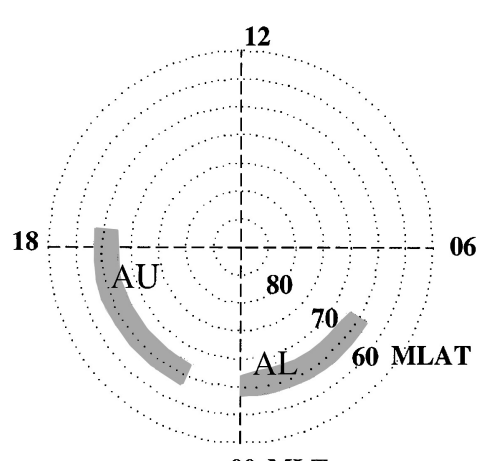

00 MLT

Fig. 11. The MLT regions (marked with gray shadings) where the local EISCAT chain gives a good estimate for the global activity (i.e. when $E_{A U}<0.2$ or $E_{A L}<0.2$ ) 
The presented results lead us to conclude that five to six evenly located meridional chains are needed for continuous monitoring of the magnetospheric substorm activity. In an ideal situation we could achieve improved $A E$ indices like in the study by Kamide et al. (1982), who use $A E_{70}$ including data from six magnetometer chains. Nevertheless, the combination of the IMAGE, CANOPUS and the Greenland chains catches a significant part of the substorms, as these stations together cover the UT-periods from 2130 to 1100. Case-studies show that the $A L$ constructed using these chains generally reproduces the real $A L$ without any significant timing delays. According to the results presented in Fig. 5, a single magnetometer chain records over-300-nT activations occurring within $\pm 5 \mathrm{~h}$ of the magnetic midnight. Thus, data from a single chain can provide information about active events for a significant portion of time.

Acknowledgements. The official AE data were kindly provided by the WDC-C2 in Kyoto and the preliminary indices for the period February-March 1991 by the Working Group on Ground-Based Observations for INTERBALL. EISCAT Magnetometer Cross was a joint German-Finnish project conducted by the Technical University of Braunschweig. The CANOPUS magnetometer data were provided by Terry Hughes and the CANOPUS instrument array was constructed and is maintained and operated by the Canadian Space Agency. We thank also Don Wallis (Herzberg Institute for Astrophysics, National Research Council, Canada) for experienced guidance in the data analysis. The data of the Greenland magnetometer network were received from Susanne Vennerström (Solar-Terrestrial Physics Division, Danish Meteorological Institute). We are obliged to Ari Viljanen for providing the program package for the analysis of the EISCAT Cross data. The work of K. K. was supported by the Academy of Finland.

Topical Editor K.-H. Glaßmeier thanks Y. Kamide and two other referees for their help in evaluating this paper.

\section{References}

Baker, K. B., and S. Wing, A new magnetic coordinate system for conjugate studies at high latitudes, J. Geophys. Res., 94, 9139-9143, 1989

Baker, D. N., S.-I. Akasofu, W. Baumjohann, J. W. Bieber, D. H Fairfield, E. W. Hones, Jr., B. Mauk, R. L. McPherron, and T. E. Moore, Substorms in the magnetosphere, in Solar Terrestrial Physics - Present and Future, NASA Publ., 1120, Chapter 8, 1984.

Baker, D. N., A. J. Klimas, R. L. McPherron, and J. Büchner, The evolution from weak to strong geomagnetic activity: an intrepretation in terms of deterministic chaos, Geophys. Res. Lett., 17, 41-44, 1990.

Bargatze, L. F., D. N. Baker, R. L. McPherron, and E. W. Hones, Jr., Magnetospheric impulse response for many levels of geomagnetic activity, J. Geophys. Res., 90, 6387-6394, 1985.
Caan, M. N., R. L. McPherron, and C. T. Russell, The statistical magnetic signature of magnetospheric substorms, Planet. Space Sci., 26, 269-279, 1978.

Davis, T. N., and M. Sugiura, Auroral electrojet activity index AE and its universal time variations, J. Geophys. Res., 71, 785-801, 1966.

Friis-Christensen, E., Y. Kamide, A. D. Richmond, and S. Matshushita, Interplanetary magnetic field control of high-latitude electric fields and currents determined from Greenland magnetometer data, J. Geophys. Res., 90, 1325-1338, 1985.

Grafe, A., Freja electron precipitation as a hint for an explosive development of the eastward electrojet, in Proceedings of the Second International Conference on Substorms, Fairbanks, Alaska, 7-11 March 1994, 391-398, 1994.

Kamide, Y., and S.-I. Akasofu, Notes on the auroral electrojet indices, Rev. Geophys. Space Phys., 21, 1647-1656, 1983.

Kamide, Y., and H. W. Kroehl, Auroral electrojet activity during isolated substorms at different local times: a statistical study, Geophys. Res. Lett., 20, 389-392, 1994.

Kamide, Y., B.-H. Ahn, S.-I. Akasofu, W. Baumjohann, E. FriisChristensen, H. W. Kroehl, H. Maurer, A. D. Richmond, G. Rostoker, R. W. Spiro, J. K. Walker, and A. N. Zeltsev, Global distribution of ionospheric and field-aligned currents during substorms as determined from six IMS meridian chains of magnetometers: initial results, J. Geophys. Res., 87, 8228-8240, 1982.

Kauristie, K., Statistical fits for auroral oval boundaries during the substorm sequence, J. Geophys. Res., 100, 21885-21895, 1995.

Lühr, H., The IMAGE Magnetometer Network, STEP Interntional, 4, 4-6, 1994.

Lühr, H., S. Thürey, and N. Klöcker, The EISCAT-magnetometer cross, technical aspects - First results, Geophys. Surv., 6, 305-315, 1984.

Pellinen, R., T. Pulkkinen, and K. Kauristie, Have we learned enough about auroral substorm morphology during the past 30 Years? in Proceedings of the Second International Conference on Substorms, Fairbanks, Alaska, 7-11 March 1994, 43-48, 1994.

Press, W. H., S. A. Teukolsky, W. T. Vetterling, and B. P. Flannery, Numerical Recipes in C, The Art of Scientific Computing, Second Edition, Cambridge University Press, Cambridge, 1992.

Rostoker, G., and T. D. Phan, Variation of auroral electrojet spatial location as a function of the level of magnetospheric acitivity, $J$. Geophys. Res., 91, 1716-1722, 1986.

Samson, J. C., T. J. Hughes, F. Creutzberg, D. D. Wallis, R. A. Greenwald, and J. M. Ruohoniemi, Observations of a detached discrete arc in association with field line resonances, J. Geophys. Res., 96, 15683-15695, 1991.

Sergeev, V. A., R. J. Pellinen, and T. I. Pulkkinen, Steady magnetospheric convection: a review of recent results, Space Sci. Rev., 75 , 551-604, 1996.

Shan, L.-H., P. Hansen, C. K. Goertz, and R. A. Smith, Chaotic appearance of the AE index, Geophys. Res. Lett., 18, 147-150, 1991.

Takalo, J., J. Timonen, and H. Koskinen, Correlation dimension and affinity of AE data and bicolored noise, Geophys. Res. Lett., 20, 1527-1530, 1993.

Weimer, D. R., Substorm time constants, J. Geophys. Res., 91, 11005-1994. 